\title{
Metrología óptica en el estudio de eventos dinámicos
}

\section{Optical metrology in the study of dynamic events}

\author{
LÓPEZ-ÁLVAREZ, Yadira Fabiola ${ }^{1,2 \uparrow^{*}}$, PEÑA-LECONA, Francisco Gerardo ${ }^{2}$, JARA-RUIZ, Ricardo ${ }^{1}$ \\ y DELGADO-GUERRERO, Sergio Humberto ${ }^{1}$
}

${ }^{1}$ Universidad Tecnológica del Norte de Aguascalientes, Estación Rincón, Rincón de Romos, Aguascalientes, 20400 México. ${ }^{2}$ Departamento de Ciencias Exactas y Tecnología, Centro Universitario de los Lagos, CULagos. Universidad de Guadalajara, Lagos de Moreno, Jalisco, 47460, México.

ID $1^{\mathrm{er}}$ Autor: Yadira Fabiola, López-Álvarez / ORC ID: 0000-0002-9041-1908, Researcher ID Thomson: T-1555-2018, CVU CONACYT ID: 375952

ID $1^{\text {er }}$ Coautor: Francisco Gerardo, Peña-Lecona / ORC ID: 0000-0002-9537-8633, CVU CONACYT ID: 122563

ID $2^{\text {do }}$ Coautor: Ricardo, Jara-Ruíz / ORC ID: 0000-0001-7725-4138, Researcher ID Thomson: T-1532-2018, CVU CONACYT ID: 630276

ID $3^{\text {er }}$ Coauthor: Sergio, Delgado-Guerrero / ORC ID: 0000-0003-2521-5887, Researcher ID Thomson: V-1747-2018, CVU CONACYT ID: 240475

DOI: $10.35429 /$ JID.2020.11.4.9.13

Recibido 23 de junio, 2020; Aceptado 26 de Diciembre, 2020

\section{Resumen}

La evolución con el tiempo de los cambios estructurales en materiales es de gran importancia en diversas áreas de la ciencia, su estudio involucra la determinación de la fase con el cambio la intensidad de las propiedades físicas a medir. En este trabajo se presenta el uso de Interferometría de Desplazamiento de Fase PSI mediante un algoritmo de combinación de cinco interferogramas o cinco pasos, los resultados se comparan con la adición de los estados estudiados en el evento dinámico, expuesto en la metodología de Interferometría Holográfica Digital DHI. La deformación fue simulada a partir de un sistema giratorio en las coordenadas $(x, y)$. Los resultados sugieren que el mapa de fase de los eventos cambiantes con el tiempo que son calculados con PSI de cinco pasos puede tener una relación con el promedio de cada punto de cambio del evento dinámico, con una diferencia de 0.96 radianes.

Metrología óptica, Eventos dinámicos, Interferometría de desplazamiento de fase

\begin{abstract}
The evolution over time of structural changes in materials is of great importance in various areas of science, its study involves determining the phase with the change in the intensity of the physical properties to be measured. In this work, the use of Phase Displacement Interferometry PSI is presented using a combination algorithm of five interferograms or five steps, the results are compared with the addition of the states studied in the dynamic event, exposed in the Digital Holographic Interferometry DHI. The deformation was simulated from a rotating system in the coordinates $(x, y)$. The results suggest that the phase map of the changing events with time that are calculated with PSI of five steps may have a relationship with the average of each change point of the dynamic event, with a difference of 0.96 radians.
\end{abstract}

Optical metrology, Dynamic events, Phase shift interferometry 


\section{Introducción}

Los avances en el estudio del comportamiento de diversas propiedades físicas han originado el uso de nuevas y mejoradas técnicas que proporcionan una medición más exacta, donde el tiempo de adquisición se ve reducido y el elemento analizado no se vea dañado, razones por las que el uso la metrología óptica ha adquirido gran aceptación dentro de diversas áreas de la ciencia (Huggins, 1992). En general, las técnicas que involucran la metrología óptica implican el uso de imágenes generadas por superposición de frentes de onda, donde la fuente tiene características de coherencia y monocromaticidad.

La representación de la superposición de frentes de onda se puede visualizar con un sensor de cámara CCD (Charge Coupled Device) a través de franjas de interferencia y su distribución geométrica es característica del elemento de estudio, este fenómeno de superposición es conocido como patrón de interferencia y representado matemáticamente como una distribución de intensidad con coordenadas espaciales $(x, y)$, ecuación (1).

$I_{i}(x, y)=A(x, y)+B(x, y) \cos \varphi(x, y)$

Donde $A, B$ y $\varphi$, son la intensidad de fondo, la modulación de la intensidad y la fase, respectivamente, esta última es la que se quiere reconstruir y la que contiene la información de los cambios del objeto de estudio. Si la intensidad muestra perturbaciones, la ecuación de intensidad de la imagen modificará el argumento coseno mediante el término $\alpha_{f}$, ecuación (2).

$I_{f}(x, y)=A(x, y)+B(x, y) \cos \left(\varphi(x, y)+\alpha_{f}\right)$

La diferencia entre los dos estados de intensidad da como resultado otro arreglo geométrico de franjas de interferencia, estas franjas son reciprocas a los dos estados de deformación, a medida que la deformación del objeto cambia, la distribución de estas franjas también lo hace. El valor absoluto de la diferencia de intensidades puede ser escrita como sigue:

$\left|I_{f}-I_{i}\right|=I_{M}\left|\cos \varphi_{f}-\cos \varphi_{i}\right|$
Entonces, para monitorear las perturbaciones del sistema, registrando el cambio de intensidad dependientes del camino óptico se analiza la distribución geométrica de las franjas de interferencia representada por los puntos de la diferencia de fase y la extracción de la fase resultante de estos dos estados de deformación dan como resultado los parámetros característicos de la medición (Gåsvik,2002). Para determinar la fase, es necesario filtrar cada interferograma de los dos estados de deformación, aplicar Trasformada de Fourier, así como operaciones entre los dos estados estudiados y finalmente la función tangente inversa y se obtiene el mapa de fase envuelto. El procedimiento para desenvolver el mapa de fase consiste en su discretización mediante el uso de método de mínimos cuadrados, el método se aplica en las componentes $(x, y)$ de la fase envuelta.

\section{Eventos dinámicos.}

Diversos enfoques se han estudiado para el análisis de cambios de fase en eventos dinámicos, uno de los más utilizados es el uso de la Interferometría Holografía Digital (DHI) de alta velocidad (Pedrini, 2007), su análisis implica el uso de la Trasformada de Fourier y filtros para obtener la fase entre dos estados que son extraídos y de su diferencia se obtiene un patrón de interferencia, $\varphi_{f-i}$, posteriormente la sumatoria en el tiempo de los cambios de fase entre la diferencia de fase de referencia $\varphi_{i}$ con cada una de las obtenidas en los distintos estados de deformación $\varphi_{f}$ y de esta manera calcular la variación física del evento dinámico (Guerrero,2016), sin embargo, esta técnica presenta la desventaja que para hologramas con alta frecuencia no es posible demodular a la información.

Otra de las técnicas para el monitoreo de eventos dinámicos es el uso de la Interferometría del Desplazamiento de Fase, esta puede mostrar gran sensibilidad a las perturbaciones ambientales, involucrando técnicas de reducción de ruido (Brock, 2005). Para determinar la distribución de fase, la interferometría de desplazamiento de fase PSI es una técnica de desenvolvimiento de mapas, donde registra una serie de interferogramas con diferencias de fase, como lo muestra la ecuación (2) y (3). 
Para realizar el proceso de la reconstrucción de la fase, en general, se aplican algoritmos con combinación de los interferogramas; el análisis se puede hacer con $n=1,2, \ldots M$ interferogramas (Schwider,1983).

$\tan \varphi=\frac{\sum_{n=1}^{M} b_{n} I_{n}}{\sum_{n=1}^{M} a_{n} I_{n}}$

Con $a_{n}$ y $b_{n}$, como coeficientes reales.

Tanto la DHI como la PSI son técnicas altamente utilizadas en el estudio de eventos dinámicos, ambas presentan limitaciones, por lo que su estudio en conjunto podría proporcionar información complementaria sobre el evento dinámico estudiado.

En este trabajo se presenta el uso de la técnica de Interferometría de Desplazamiento de Fase PSI para el estudio de eventos dinámicos, se realiza la demodulación de franjas de interferencia entre los estados de deformación mediante el algoritmo expuesto por Schwider et al (Schwider,1983) de cinco pasos y se comparan los resultados de la fase total del evento a través de una sumatoria de las diferencias de fases calculadas en cada punto de la deformación, según la técnica DHI.

El objetivo es demostrar que es factible determinar la fase de todo el evento, analizando su evolución como una sumatoria de los cambios presentados y al comparar la fase resultante con Interferometría de Desplazamiento de Fase, los resultados no distan de ser similares.

\section{Desarrollo y resultados}

Para implementar la técnica de desplazamiento de fase en el evento dinámico, se simularon las franjas de interferencia originadas por la deformación $\alpha_{f}$, según se indica en la ecuación (2).

$\alpha_{f}=x \cos \theta-y \sin \theta$

Para $\theta=0, \frac{\pi}{2}, \pi, \frac{3}{2} \pi, 2 \pi, \quad$ obteniendo cinco patrones de franjas de interferencia, estos patrones de interferencia son el resultado de la diferencia de dos intensidades con diferentes estados de deformación.

$$
\text { Para:(a) } \cos \varphi(x, y)-\cos (\theta=0) \text {, }
$$

(b) $\cos \varphi(x, y)-\cos \left(\theta=\frac{\pi}{2}\right)$, (c) $\cos \varphi(x, y)-$ $\cos (\theta=\pi),(\mathrm{d})=\cos \varphi(x, y)-\cos \left(\theta=\frac{3}{2} \pi\right) \mathrm{y}$ (e) $\cos \varphi(x, y)-\cos (\theta=2 \pi)$, Figura 1 .

Para determinar la fase de cada uno de los interferogramas en el evento dinámico se recurre al uso de la Transformada de Fourier directa e inversa, el uso de filtros y la función tangente inversa, ecuaciones (6-9).

$$
\begin{aligned}
& F\left\{\left(I_{N}-I_{0}\right)\right\} \\
& w=e^{-a\left(x^{2}+y^{2}\right)^{2}} \\
& F^{-1}\left(F\left\{\left(I_{N}-I_{0}\right)\right\} * w\right) \\
& \varphi_{w}=\tan ^{-1}\left(F^{-1}\left(F\left\{\left(I_{N}-I_{0}\right)\right\} * w\right)\right.
\end{aligned}
$$

Para encontrar la fase del evento es necesario aplicar algoritmos de desenvolvimiento mediante la discretización de los resultados, la Figura 2 muestra una comparativa entre las fases de cada cambio de intensidad.

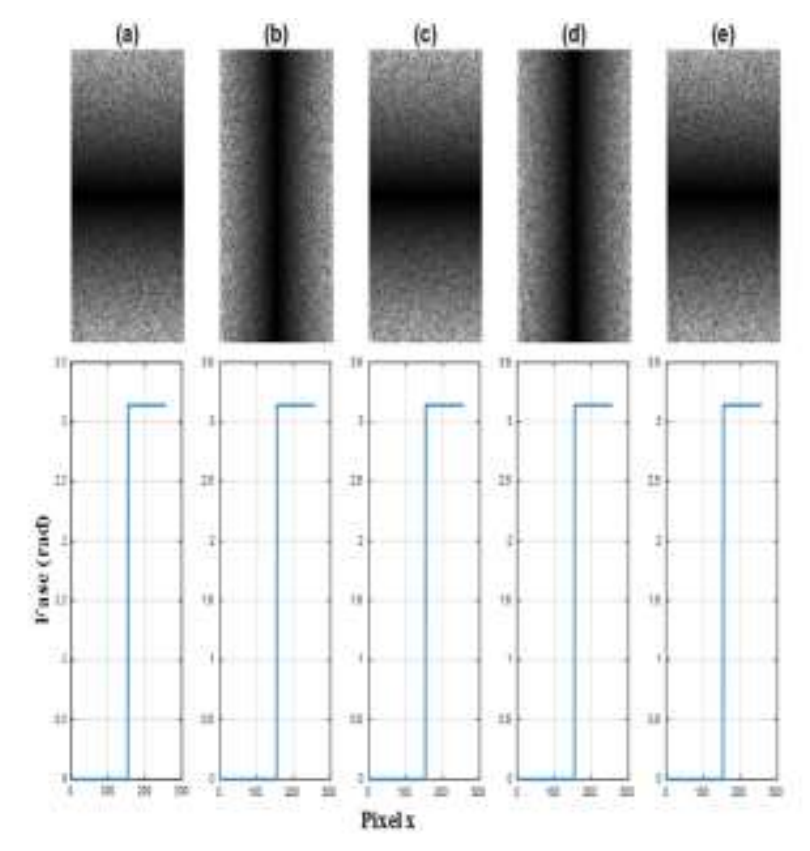

Figura 1 Patrón y mapa de fase de franjas de interferencia, (a) $\theta=0$, (b) $\theta=\pi / 2$, (c) $\pi$, (d), $3 / 2 \pi$. (e) $2 \pi$ Fuente: Elaboración Propia 


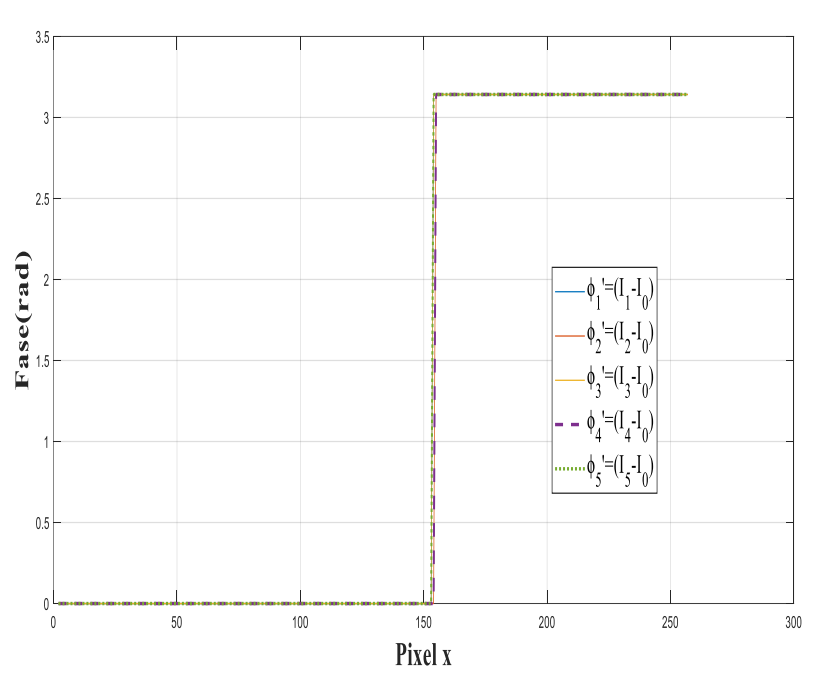

Figura 2 Comparativa de mapas de diferencia de fase de franjas de interferencia, (a) $\theta=0$, (b) $\theta=\frac{\pi}{2}$, (c) $\pi$, (d),$\frac{3}{2} \pi$. (e) $2 \pi$

Fuente: Elaboración Propia

La Figura 3, muestra el comportamiento de los mapas de fase, $\phi_{1}$ representa la sumatoria de cada una de las fases que componen el evento dinámico, mientras que $\phi_{2}$ se obtiene utilizando el algoritmo de cinco pasos cuando la fase cambia en intervalos de $\pi / 2$. La mayor diferencia se presenta en la región central del eje $x$ de ambos cálculos, sin embargo, el comportamiento del mapa de fase se sigue conservando, así como los valores de la fase.

Los algoritmos utilizados para determinar las fases resultantes se muestran en las ecuaciones (10) y (11).

La ecuación (10), muestra el algoritmo propuesto como una sumatoria de los cambios que componen al evento dinámico total, $N$ representa el total de estudios holográficos que se tomaron con DHI, este puede ser calculado con dos hologramas, ya que es indispensable obtener una diferencia entre las dos fases resultantes, en este trabajo no se presenta la demodulación de los cambios del evento con DHI ya que el objetivo radica en demostrar el comportamiento de los mapas de fase.

Por su parte la ecuación (11) representa el algoritmo de cinco pasos, expuesto por Schwider et al, este algoritmo es calculado mediante la combinación de interferogramas que representan los cambios en el evento dinámico.

$\phi_{1}=\sum_{I=0}^{N} \frac{\left(I_{N}-I_{0}\right)}{N}$

$$
\phi_{2}=\frac{2\left(I_{5}-I_{1}\right)}{I_{4}+I_{0}-2\left(I_{2}\right)}
$$

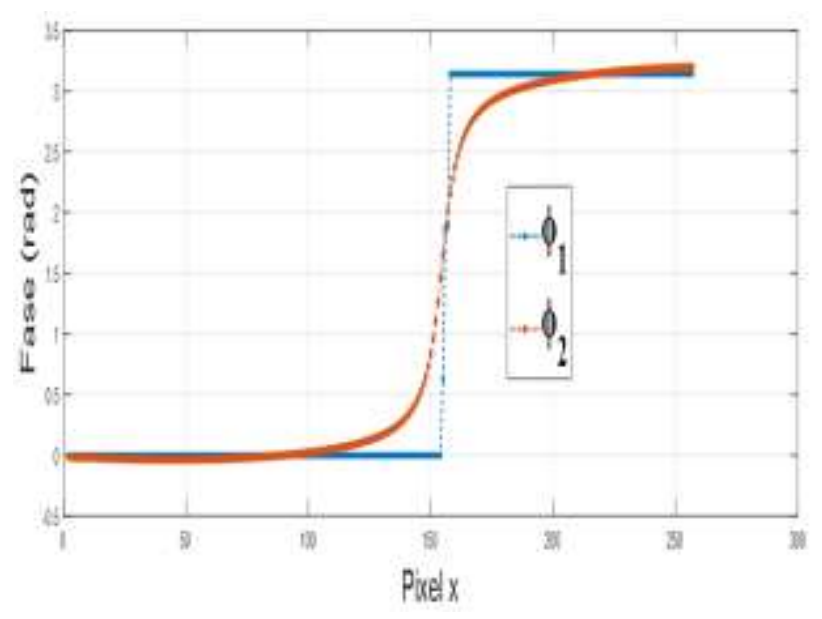

Figura 3 Comparativa de mapas de fase utilizando técnicas ópticas: PSI y adición de cambios en eventos dinámicos DHI

Fuente: Elaboración Propia

\section{Conclusiones}

Se presenta la comparativa entre dos técnicas de metrología óptica para demostrar la similitud del comportamiento de los mapas de fase resultado de un evento dinámico. Se hace uso de la Interferometría de Desplazamiento de Fase con el algoritmo de cinco pasos para calcular el mapa de fase total y el resultado se compara con la sumatoria de cada uno de los estados, concepto expuesto en DHI. Con valor promedio del evento se sugieren que existe una similitud en el comportamiento del mapa de fase utilizando PSI y DHI, la diferencia entre los mapas de fase determinados es de 0.96 radianes, en el centro del eje $\mathrm{x}$.

\section{References}

Gåsvik Kjell J. Optical Metrology, John Wiley \& Sons Ltd, 3ed, 316 (2002).

Guerrero-Méndez, C., Anaya, T. S., AraizaEsquivel, M., Balderas-Navarro, R. E., ArandaEspinoza, S., López-Martínez, A., \& OlveraOlvera, C. (2016). Real-time measurement of the average temperature profiles in liquid cooling using digital holographic interferometry. Optical Engineering, 55(12), 121730.

Huggins, R. W., Abbas, G. L., Hong, C. S., Miller, G. E., Porter, C. R., \& Van Deventer, B. Fiber-coupled position sensors for aerospace applications. Optics and Lasers in Engineering, 16(2-3), 79-103, (1992).

LÓPEZ-ÁLVAREZ, Yadira Fabiola, PEÑA-LECONA, Francisco Gerardo, JARA-RUIZ, Ricardo y DELGADO-GUERRERO, Sergio Humberto. Metrología óptica en el estudio de eventos dinámicos. Revista del Diseño Innovativo. 2020 
Neal Brock, John Hayes, Brad Kimbrough, James Millerd, Michael North-Morris, Matt Novak, and James C. Wyant "Dynamic interferometry", Proc. SPIE 5875, Novel Optical Systems Design and Optimization VIII, 58750F (29 August 2005)).

Pedrini, G., \& Osten, W. (2007). Time resolved digital holographic interferometry for investigations of dynamical events. In Optics InfoBase Conference Papers. Optical Society of America (OSA).

Schwider J., Burow R., Elssner K.-E., Grzanna J., Spolaczyk R., and Merkel K., "Digital wavefront measuring interferometry: some systematic error sources," Appl. Opt. 22, 34213432 (1983). 